\title{
On Some New Classes of Pseudo-Differential Operators
}

\author{
Vladimir Vasilyev \\ Chair of Differential Equations, Belgorod National Research University, Belgorod, 308007, Russia \\ vladimir.b.vasilyev@gmail.com
}

\begin{abstract}
We study Fredholm properties for a special class of elliptic pseudo-differential operators. Using a local principle we give boundedness theorems for such operators and describe their Fredholm properties in Sobolev-Slobodetskii spaces of a variable order. For a half-space case we introduce a certain operator family which helps describing Fredholm properties.
\end{abstract}

\section{INTRODUCTION}

Pseudo-differential operators and equations were born in past century (see, for example, [1-3]) and now this theory lives and develops. In this paper we would like to enlarge class of spaces of spaces in which such pseudo-differential operators can act, and describe their Fredholm properties in these spaces. We use spaces of variable order and consider also pseudo-differential operators of variable order. First similar constructions were introduced in [4] (and further there were some generalizations and refinements), but our considerations contain new results related to boundedness and Fredholm properties and based on a local principle. Moreover we study a family of spaces of a variable order and intend to apply this methodology to appearing boundary value problems. Also we hope these consideration will be useful for more complicated situations when we deal with a cone instead of a half-space [5].

\section{Local Sobolev-Slobodetskii Spaces}

Let $s: \mathbb{R}^{m} \rightarrow \mathbb{R}$ be an arbitrary function satisfying the following conditions:

- the finite limit $\lim _{|x| \rightarrow+\infty} s(x)$ exists,

- the function $s(x)$ satisfies the Lipschitz condition in $\mathbb{R}^{m}$, i. e. there is a positive constant $C>0$ such that

$$
\left|s\left(x_{1}\right)-s\left(x_{2}\right)\right| \leq c\left|x_{1}-x_{2}\right|, \quad \forall x_{1}, x_{2} \in \mathbb{R}^{m} .
$$

For fixed $x \in \mathbb{R}^{m}$ we introduce the following definition.

Definition 1 By definition a local Sobolev-Slobodetskii space $H^{(x)}\left(\mathbb{R}^{m}\right)$ consists of distributions with finite value

$$
\|u\|_{s(x)} \equiv\left(\int_{\mathbb{R}^{u}}(1+|\xi|)^{2 s(x)}|\tilde{u}(\xi)|^{2} d \xi\right)^{1 / 2},
$$

where $\bar{u}$ denotes the Fourier transform of the function $u$.

The value $\|u\|_{s(x)}$ is called a local $H^{s}$-norm of the function $u$.

For a brevity we will write $H^{s(x)}$ instead of $H^{s(x)}\left(\mathbb{R}^{m}\right)$, and if we speak on $H^{s(x)}$-functions with supports in a certain domain $D \subset \mathbb{R}^{m}$ then we write $H^{s(x)}(D)$. 
Definition 2 The universal Sobolev-Slobodetskii super-space $H^{S M}$ is called the space which includes all local Sobolev-Slobodetskii spaces with finite norm

$$
\left\|\left.u\right|_{S_{M}}=\sup _{x \in \mathbb{R}^{m}}\right\| u\|\|_{s(x)} .
$$

The universal Sobolev-Slobodetskii subspace $H^{s_{1}}$ is called the space which is inside of all local SobolevSlobodetskii spaces with finite norm

$$
\|u\|_{s_{m}}=\inf _{x \in \mathbb{R}^{n^{2}}}\|u\|_{s(x)} .
$$

Obviously,

$$
\|u\|_{S_{m}} \leq\|u\|_{s(x)} \leq\|u\|_{S_{M}}, \quad \forall X \in \mathbb{R}^{m} .
$$

Let us denote $S\left(\mathbb{R}^{m}\right)$ the Schwartz class of infinitely differentiable rapidly decreasing at infinity functions; this class is dense in each local Sobolev-Slobodetskii space [3].

Lemma $1 \quad$ For $u \in S\left(\mathbb{R}^{m}\right)$ we have estimates

for a certain $l \in\left[s_{1}, s_{2}\right]$.

$$
\begin{gathered}
\left|(1+\mid \xi)^{s_{1}}-(1+|\xi|)^{s_{2}}\right| \leq c_{1}\left|s_{1}-s_{2}\right| \cdot(1+|\xi|)^{I}, \\
\left|\|u\|_{s_{\left(x_{1}\right)}}-\|u\|_{s\left(x_{2}\right)}\right| \leq c_{2}\left|x_{1}-x_{2}\right| \cdot\|u\|_{s_{M}}
\end{gathered}
$$

Let $H_{X}$ be a family of Hilbert spaces parametrized by points $x \in \mathbb{R}^{m}$. We denote $\|u\|_{X}$ the norm of element $u \in H^{s(x)}$ and assume that $S$ is a dense subset in $H^{s(x)}, \forall x \in \mathbb{R}^{m}$. For $u \in S$ we define the functional

$$
f(x, u)=\|u\|_{X} .
$$

Definition 3 We say that a family of Hilbert spaces $\left\{H_{x}\right\}_{x \in \mathbb{R}^{m}}$ is a local continuous family in the point $x_{0} \in \mathbb{R}^{m}$ if for fixed $u \in S$ the functional $f(x, u)$ is continuous in the point $X_{0}$.

Lemma 2 The family $H^{(5)}$ is a local continuous family.

\section{OPERATORS OF A VARIABLE ORDER}

Definition 4 Given function $A(x, \xi)$ defined in $\mathbb{R}^{m} \times \mathbb{R}^{m}$ a pseudo-differential operator $A$ is called an operator of the following type

$$
(A u)(x)=\iint_{\mathbb{R}^{m}} \int_{\mathbb{R}^{m}} A(x, \xi) e^{i(x-y) \xi} u(y) d y d \xi, \quad x \in \mathbb{R}^{m} .
$$

The function $A(x, \xi)$ is called a symbol of the operator $A$.

Let $\alpha: \mathbb{R}^{m} \rightarrow \mathbb{R}$ be a function with the same properties as $s(x)$.

Definition 5 The class $E_{\alpha(x)}$ consists of functions $A(x, \xi)$ defined in $\mathbb{R}^{m} \times \mathbb{R}^{m}$ and satisfying the conditions

$$
c_{1}(1+\mid \xi)^{\alpha(x)} \leq|A(x, \xi)| \leq c_{2}(1+|\xi|)^{\alpha(x)}
$$

and for each point $x_{0} \in \mathbb{R}^{m}$ there exists a neighborhood $U_{x_{0}}$ such that for all $x \in U_{x_{0}}$ the following inequality

$$
\left|A(x, \xi)-A\left(x_{0}, \xi\right)\right| \leq c_{3}\left|x-x_{0}\right|(1+\mid \xi)^{\alpha(x)}
$$

holds, where $c_{1}, c_{2}, c_{3}$ are positive constants. The function $\alpha: \mathbb{R}^{m} \rightarrow \mathbb{R}$ is called a variable order of a pseudo differential operator (symbol).

If $x_{0}$ is an infinity then we need to require satisfying the following inequality

$$
|A(x, \xi)-A(\infty, \xi)| \leq c_{4}|x|^{-1}(1+|\xi|)^{\alpha(x)},
$$

instead of the inequality (3).

Example. A very simple example is so called fractional Laplacian of a variable order. Its symbol is

$$
A(x, \xi)=\left(1+\xi_{1}^{2}+\xi_{2}^{2}+\cdots+\xi_{m}^{2}\right)^{\alpha(x)} .
$$

All conditions mentioned above are satisfied. 


\section{Boundedness Theorems}

If we fix $x_{0} \in \mathbb{R}^{m}$ then the operator $A_{x_{0}}$ is an operator with the symbol $A\left(x_{0}, \xi\right)$.

Lemma 3 If $A\left(x_{0}, \xi\right) \in E_{\alpha\left(x_{0}\right)}$ then the operator $A_{x_{0}}$ is bounded in local Sobolev-Slobodetskii space, $A_{x_{0}}: H^{s\left(x_{0}\right)} \rightarrow$ $I I^{s\left(x_{0}\right)-\alpha\left(x_{0}\right)}$.

This property is called a local boundedness.

Definition 6 An operator $A$ with the symbol $A(x, \xi)$ is called a local bounded operator if for each point $x_{0} \in \mathbb{R}^{m}$ the operator $A_{x_{0}}: H^{s\left(x_{0}\right)} \rightarrow H^{s\left(x_{0}\right)-\alpha\left(x_{0}\right)}$ with the symbol $A\left(x_{0}, \xi\right)$ is bounded.

Theorem $4 \quad$ If $A(x, \xi) \in E_{\alpha(x)}$ then the function $x_{0} \longmapsto\left\|A_{x_{0}}\right\|_{s\left(x_{0}\right)}$ is continuous at each point $x_{0} \in \mathbb{R}^{m}$ including the infinity.

Remark 1 It means the property of a local boundedness is conserved in enough small neighborhood.

Theorem 5 If an operator $A$ with the symbol $A(x, \xi)$ is local bounded then it is bounded $A: H^{S_{M}} \rightarrow H^{(s-\alpha)_{m}}$.

Remark 2 It seems it is enough to require a local boundedness on a certain countable set including the infinity.

\section{Compact Operators in Spaces $H^{s(x)}$}

Let $\left\{T_{x}: H^{s_{1}(x)} \rightarrow H^{S_{2}(x)}\right\}_{x \in \mathbb{R}^{m}}$ be a family of linear bounded operators.

Lemma 6 If the linear bounded operator $T_{x_{0}}: H^{s_{1}\left(x_{0}\right)} \rightarrow H^{s_{2}\left(x_{0}\right)}$ is compact then there is a neighborhood $U_{x_{0}}$ of the point $x_{0}$ such that for all $x_{1} \in U_{x_{0}}$ the operator $T_{x_{1}}: H^{s_{1}\left(x_{1}\right)} \rightarrow H^{s_{2}\left(x_{1}\right)}$ is compact.

Let $\psi_{x_{0}}(x) \in C_{0}^{\circ \circ}\left(\mathbb{R}^{m}\right)$ be a function equals to 1 in some neighborhood of the point $x_{0}$.

Corollary $7 \quad$ An operator with the symbol $\psi_{x_{0}}(x) A(x, \xi)-A\left(x_{0}, \xi\right)$ is compact in some neighborhood of the point $x_{0}$ as operator $H^{s(x)} \rightarrow H^{s(x)-\alpha(x)}$.

\section{Fredholmness}

Definition $7 \quad$ An operator $A_{x_{0}}$ with the symbol $A\left(x_{0}, \xi\right)\left(x_{0} \in \mathbb{R}^{m}\right.$ is fixed) we call the local representative of the operator $A$ in the point $x_{0}$.

Theorem 8 If local representatives $A_{x_{0}}: H^{s\left(x_{0}\right)} \rightarrow H^{s\left(x_{0}\right)-\alpha\left(x_{0}\right)}$ of the operator $A$ are invertible at each point of $\mathbb{R}^{m}$ including the infinity then the operator $A: H^{S_{M}} \rightarrow H^{(s-\alpha)_{m}}$ has a Fredholm property.

Remark 3 It is enough to require an invertibility for the local representatives in a certain dense set.

\section{A HALF-SPACE CASE}

Definition 8 Let $A(x, \xi)$ be a function defined in $\mathbb{R}_{+}^{m} \times \mathbb{R}^{m}$. A pseudo-differential operatorA in a half-space with the symbol $A(x, \xi)$ is called an operator of the following type

$$
(A u)(x)=\int_{\mathbb{R}_{+}^{m}}\left(\int_{\mathbb{R}^{m}} A(x, \xi) e^{j(x-y) \xi} u(y) d \xi\right) d y, \quad x \in \mathbb{R}_{+}^{m} .
$$

We consider the following equation in a half-space

$$
(A u)(x)=v(x), \quad x \in \mathbb{R}_{+}^{m},
$$

related to such an operator. 
According to [3] we introduce the space $H^{s(x)}\left(\mathbb{R}_{+}^{m}\right)$ of functions from $H^{s(x)}\left(\mathbb{R}^{m}\right)$ with support in $\overline{\mathbb{R}_{+}^{m}}$, this is space of solutions, and the space $H_{0}^{\mathrm{s}(x)}\left(\mathbb{R}_{+}^{m}\right)$ of functions (distributions) from $S^{\prime}\left(\mathbb{R}^{m}\right)$ with suppoit in $\overline{\mathbb{R}_{+}^{m}}$, these must admit analytical continuation in a whole $H^{s(x)}\left(\mathbb{R}^{m}\right)$ with finite norm

$$
\|v\|_{s(x)}^{+}=\inf \|\ell \eta\|_{s(x)},
$$

where infimum is taken over all continuations $\ell$. tatives.

For studying a Fredholmness of the equation (6) we use a local principle. We extract two types of local represen-

1) For inner point $x_{0} \in \mathbb{R}_{+}^{m}$ this is well-known operator

$$
\left(A_{N 0} u\right)(x)=\int_{\mathbb{R}^{m}} \int_{\mathbb{R}^{m}} A\left(x_{0}, \xi\right) e^{i(x-y) \xi} u(y) d y d \xi, \quad x \in \mathbb{R}^{m} .
$$

2) For boundary point $x_{0}^{\prime}=\left(x_{0}^{\prime}, 0\right) \in \mathbb{R}^{m-1}$ (we use notation $x_{0}=\left(x_{0}^{\prime}, x_{m}^{(0)}\right)$ for the $x_{0} \in \mathbb{R}^{m}$ ) it will be the operator

$$
\left(A_{x_{0}} u\right)(x) \longmapsto \int_{\mathbb{R}_{+}^{m}}\left(\int_{\mathbb{R}^{m}} A\left(x_{0}^{\prime}, \xi\right) e^{i(x-y) \xi} u(y) d \xi\right) d y, \quad x \in \mathbb{R}_{+}^{m} .
$$

Thus, we have two operator families.

Theorem 9 If all operators of the family $A_{x_{0}^{\prime}}: H^{s\left(x^{x}\right)}\left(\mathbb{R}_{+}^{m}\right) \rightarrow H^{s\left(x^{\prime}\right)-\alpha\left(x^{\prime}\right)}\left(\mathbb{R}_{+}^{m}\right)$ are bounded then the operator (5) is bounded as operator $H^{S_{M}}\left(\mathbb{R}_{+}^{m}\right) \rightarrow H^{(s-\alpha)_{m}}\left(\mathbb{R}_{+}^{m}\right)$.

If all operators of two operator family are invertible then the operator (5) is Fredholm operator as operator $H^{s_{M}}\left(\mathbb{R}_{+}^{m}\right) \rightarrow H^{(s-\alpha)_{m}}\left(\mathbb{R}_{+}^{m}\right)$.

\section{Variable Index of Factorization}

This is key point for studying pseudo-differential equations in a half-space. We will give a corresponding definition and show its applicability to the equation (7) with fixed $x^{\prime} \in \mathbb{R}^{m-1}$.

Definition 9 Factorization of the symbol $A\left(x^{\prime}, \xi\right)$ on a variable $\xi_{m}$ is called its representation in the form

$$
A\left(x^{\prime}, \xi^{\prime}, \xi_{m}\right)=A_{+}\left(x^{\prime}, \xi^{\prime}, \xi_{m}\right) A_{-}\left(x^{\prime}, \xi^{\prime}, \xi_{m}\right),
$$

where the factors $A_{+}\left(A_{-}\right)$admit analytical continuation on $\xi_{m}$ in upper (lower) complex half-plane $\xi_{m} \pm i \tau, \tau>0$, under almost all $\xi^{\prime} \in \mathbb{R}^{m-1}$ and satisfy estimates

$$
\begin{gathered}
\left|A_{+}\left(x^{\prime}, \xi^{\prime}, \xi_{m}\right)\right| \leq c_{1}\left(1+\left|\xi^{\prime}\right|+\left|\xi_{m}\right|+|\tau|\right)^{æ\left(x^{\prime}\right)} \\
\left|A_{-}\left(x^{\prime}, \xi^{\prime}, \xi_{m}\right)\right| \leq c_{1}\left(1+\left|\xi^{\prime}\right|+\left|\xi_{m}\right|+|\tau|\right)^{\alpha\left(x^{\prime}\right)-æ\left(x^{\prime}\right)}, \quad \forall \tau \in \mathbb{R} .
\end{gathered}
$$

The function $æ\left(x^{\prime}\right), x^{\prime} \in \mathbb{R}^{m-1}$, is called a variable index of factorization.

Let us note that such factorization can be constructed effectively with a help of the Cauchy type integral [3].

Below we will assume that the function $æ\left(x^{\prime}\right)$ has the same properties as $s\left(x^{\prime}\right)$.

\section{A Local Solvability and Boundary Conditions}

Now there is a question how one can use the theorem 9. In other words we need conditions which guarantee an invertibility for local representatives of boundary operator.

Theorem 10 Let

$$
\left|æ\left(x^{\prime}\right)-s\left(x^{\prime}\right)\right|<1 / 2, \quad \forall x^{\prime} \in \mathbb{R}^{m-1} .
$$

Then the operator (7) is invertible as an operator $H^{s\left(x^{\prime}\right)}\left(\mathbb{R}_{+}^{m}\right) \rightarrow H^{s\left(x^{\prime}\right)-\alpha\left(x^{\prime}\right)}\left(\mathbb{R}_{+}^{m}\right)$ under each fixed $x^{\prime}$. 
Collecting the theorems 9, 10 we obtain a Fredholm property for pseudo-differential operator (equation) in a half-space.

Theorem $11 \quad$ Let $A(x, \xi) \in E_{\alpha(x)}$. If

$$
\left|æ\left(x^{\prime}\right)-s\left(x^{\prime}\right)\right|<1 / 2, \quad \forall x^{\prime} \in \mathbb{R}^{m-1}, \quad \forall x^{\prime} \in R^{m-1},
$$

then the operator (5) has a Fredholm property as an operator $H^{S_{M}}\left(\mathbb{R}_{+}^{m}\right) \rightarrow H^{(s-\alpha)_{m}}\left(\mathbb{R}_{+}^{m}\right)$.

There are situations for which the condition (8) does not hold. We consider here one of possible variants.

Let $x\left(x^{\prime}\right)-s\left(x^{\prime}\right)=m+\delta,|\delta|<1 / 2, m \in \mathbb{N}, \forall x^{\prime} \in \mathbb{R}^{m-1}$. Now for fixed $x^{\prime}$ even the operator (7) is non-invertible, but we know a general solution of corresponding equation [3]. We will briefly describe its form to explain appearing boundary operators.

Thus, we consider an equation with the operator $(7)$ and right-hand side $f \in H_{0}^{s\left(x_{0}^{\prime}\right)-\alpha\left(x_{0}^{\prime}\right)}\left(\mathbb{R}_{+}^{m}\right)$.

We will remind that for fixed $x_{0}^{\prime} \in \mathbb{R}^{m-1}$ a general solution of the equation

$$
\left(A_{x_{0}} u\right)(x)=f(x), \quad x \in \mathbb{R}_{+}^{m}
$$

with the operator (7) in Fourier image has the following form [3]

$$
\tilde{u}(\xi)=A_{+}^{-1}\left(x_{0}^{\prime}, \xi\right) P_{m}(\xi) \Pi_{+} P_{m}^{-1}(\xi) A_{-}^{-1}\left(x_{0}^{\prime}, \xi\right) \widetilde{\ell f}(\xi)+A_{+}^{-1}\left(x_{0}^{\prime}, \xi\right) \sum_{k=1}^{m} \bar{c}_{k}\left(x_{0}^{\prime}, \xi^{\prime}\right) \xi_{m}^{k-1},
$$

where the operator $\Pi_{+}$is the Cauchy type integral

$$
\left(\Pi_{+} \tilde{u}\right)(\xi)=\frac{1}{2 \pi} \lim _{\tau \rightarrow 0+} \int_{-\infty}^{+\infty} \frac{\tilde{u}\left(\xi^{\prime} \cdot \eta_{m}\right) d \eta_{m}}{\xi_{m}-\eta_{m}+\dot{i} \tau} d d \eta_{m}
$$

$P_{m}(\xi) \in E_{m}$ is an arbitrary polynomial, $c_{k}\left(x_{0}^{\prime}, \xi\right)$ are arbitrary functions from $H^{s_{k}\left(x_{0}^{\prime}\right)}\left(\mathbb{R}^{m-1}\right), s_{k}\left(x_{0}^{\prime}\right)=s\left(x_{0}^{\prime}\right)-æ\left(x_{0}^{\prime}\right)+$ $k-1 / 2, k=1, \cdots, m$.

To determine uniquely $c_{k}$ we choose $m$ bounded pseudo-differential operators $B_{j}: H^{s\left(x_{0}^{\prime}\right)}\left(\mathbb{R}_{+}^{m}\right) \rightarrow H^{s\left(x_{0}^{\prime}\right)-\alpha_{j}\left(x_{0}^{\prime}\right)}\left(\mathbb{R}_{+}^{m)}\right.$ with symbols $B_{j}\left(x_{0}, \xi\right), j=1, \cdots, m$, and $\gamma$ is a restriction operator on the hyperplane $x_{m}=0$ so that operators $\gamma B_{j}$ are bounded $H^{s\left(x_{0}^{\prime}\right)}\left(\mathbb{R}_{+}^{m}\right) \rightarrow H^{s\left(x_{0}^{\prime}\right)-\alpha_{j}\left(x_{0}^{\prime}\right)-1 / 2}\left(\mathbb{R}^{m-1}\right)$ under the condition $s\left(x_{0}^{\prime}\right)-\alpha_{j}\left(x_{0}^{\prime}\right)-1 / 2>0, \forall x_{0}^{\prime} \in \mathbb{R}^{m-1}$. Let us introduce a new local sobolev-Slobodetskii space as a direct sum

$$
H^{s_{\alpha}\left(x_{0}^{\prime}\right)} \equiv H^{s\left(x_{0}^{\prime}\right)}\left(\mathbb{R}_{+}^{m}\right) \oplus \sum_{j=1}^{m} H^{s\left(x_{0}^{\prime}\right)-\alpha_{j}\left(x_{0}^{\prime}\right)-1 / 2}\left(\mathbb{R}^{m-1}\right)
$$

For the point $x_{0}^{\prime} \in \mathbb{R}^{m-1}$ we introduce a local operator $H^{s\left(x_{0}^{\prime}\right)}\left(\mathbb{R}_{+}^{m}\right) \rightarrow H^{s_{\alpha}\left(x_{0}^{\prime}\right)}$ by the formula

$$
B_{x_{0}^{\prime}} u=\left(A_{x_{0}^{\prime}} u, \gamma B_{1} u, \gamma B_{1} u \cdots, \gamma B_{m} u\right)
$$

Therefore, the operator $B_{x_{0}}$ is given by pasting $m$ additional operators together with a family of additional spaces to the operator $A_{x_{0}}$ at each point $x_{0}^{\prime} \in \mathbb{R}^{m-1}$.

We can conclude as above that under our assumptions all such operators are locally bounded, and it implies a boundedness of the $(10)$.

One can study an invertibility of the local operator (10) by the Fourier transform, it permits to reduce an identification problem for the functions $c_{k}$ to unique solvability of some $m \times m$-system of linear algebraic equations. Non-vanishing for a determinant of the latter system is necessary and sufficient condition for an invertibility of the local operator $B_{X_{0}}$.

Remark 4 The left case $æ\left(x^{\prime}\right)-s\left(x^{\prime}\right)=-m+\delta,|\delta|<1 / 2, m \in \mathbb{N}, \forall x^{\prime} \in \mathbb{R}^{m-1}$, can dt considered analogously. For this case we extend the domain of $A_{X^{\prime}}$, and we past to the domain additional local Sobolev-Slobodetskii spaces with additional unknowns in these spaces. 


\section{CONCLUSION}

It seems a lot of results obtained by a local principle can be transferred on operators and equations of a variable order. In our opinion more interesting case is studying such operators and equations on manifolds with non-smooth boundaries like [5-9].

\section{ACKNOWLEDGMENTS}

This work was supported by the State contract of the Russian Ministry of Education and Science (contract No 1.7311.2017/8.9).

\section{REFERENCES}

[1] F. Treves, Introduction to Pseudodifferential Operators and Fourier Integral Operators (Springer, New York, 1980).

[2] M. Taylor, Pseudodifferential Operators (Princeton University Press, Princeton, 1981).

[3] G. Eskin, Boundary Value Problems for Elliptic Pseudodifferential Equations (AMS, Providence, 1981).

[4] M. I. Vishik and G. I. Eskin, Russ. Math. Surv. 22, 13-75 (1967).

[5] V. B. Vasil'ev, Wave Factorization of Elliptic Symbols: Theory andApplications. Introduction to the Theory of Boundary Value Problems in Non-Smooth Domains (Kluwer Academic Publishers, Dordrecht-BostonLondon, 2000).

[6] V. B. Vasil'ev, Differ. Equ. 51, 1113-1125 (2015).

[7] V. B. Vasil'ev, J. Math. Sci. 230, 175-183 (2018).

[8] V. B. Vasilyev, "Pseudo-differential operators on manifolds with a singular boundary," in Modern Problems in Applied Analysis, edited by P. Drygas and S. Rogosin (Birkhauser/Springer, Cham, 2018), pp. 169-179.

[9] V. B. Vasilyev, "Elliptic equations, manifolds with non-smooth boundaries, and boundary value problems," in New Trends in Analysis and Interdisciplinary Applications. Selected Contributions of the 10th ISAAC Congress, Macau 2015, edited by P. Dang, M. Ku, T. Qian, and L. G. Rodino (Birkhauser/Springer, Cham, 2017), pp. 337-344. 